\title{
Radio holographic principle for observing natural processes in the atmosphere and retrieving meteorological parameters from radio occultation data
}

\author{
K. Igarashi' ${ }^{1}$, A. Pavelyev², K. Hocke ${ }^{3}$ D. Pavelyev², I. A. Kucherjavenkov², S. Matyugov², A. Zakharov², and O. Yakovlev² \\ ${ }^{1}$ Communications Research Laboratory, Ministry of Posts and Telecommunications, 4-2-1 Nukui-Kita Machi, Koganei-shi, Tokyo 184-0015, Japan \\ ${ }^{2}$ Institute of Radio Engineering and Electronics of Russian Academy of Sciencies (IRE RAS), \\ Fryazino, Vvedenskogo sq. 1, 141120 Moscow region, Russia \\ ${ }^{3}$ GeoForschungsZentrum Potsdam (GFZ-Potsdam), Telegrafenberg, 14473 Potsdam, Germany
}

(Received December 20, 1999; Revised September 25, 2000; Accepted September 26, 2000)

\begin{abstract}
The radio holographic principle is briefly described and tested by using radio occultation data of the GPS/MET and MIR/GEO experiments. Sub-Fresnel spatial resolution $\sim 12 \mathrm{~m} /$ pixel was achieved using focused synthetic aperture radio holographic approach, and direct evidence of multibeam propagation effects in the atmosphere was obtained. The achieved instrumental accuracy in angular distance measurements was near 0.004 milliradian/pixel, and observed angular distance between different rays was equal to 0.3 milliradians. The angular resolution of the radio holographic method depends on the wavelength as $\lambda^{1}$ compared to $\lambda^{1 / 2}$ in conventional methods. In general case the principal limit of the vertical resolution may be determined using focused synthetic aperture antenna theory and may achieve a value $\sim 20-40 \mathrm{~m}$ under assumptions of spherical symmetry and quiet atmospheric conditions. Wave structures were discovered in the altitude distribution of the gradient electron density at a height interval of 60-95 km with spatial period 1-2 km and vertical resolution 300-500 m. Good correspondence was found between the temperature profiles revealed by radio holographic analysis and those obtained by traditional retrieval using UCAR GPS/MET data.
\end{abstract}

\section{Introduction}

The high accuracy of the radio occultation sounding was demonstrated using the Global Positioning System (GPS) at wavelengths 19 and $24 \mathrm{~cm}$ (Melbourne et al., 1994; Ware et al., 1996; Kursinski et al., 1996, 1997; Rocken et al., 1997; Feng and Herman, 1999; Schreiner et al., 1999). First experiments in another frequency bands have been provided in 1989-1998 years at wavelengths 2 and $32 \mathrm{~cm}$ as described by Yakovlev et al. (1995) and Yakovlev (1998).

Now the first stage of investigating of the Earth's atmosphere and ionosphere has been accomplished using this method. The results of this stage of radio occultation investigations show the need to design a new radio occultation methodology that includes combined amplitude and phase data analysis for achieving better spatial resolution and accuracy in vertical profiles of atmospheric and ionospheric parameters. Some basic approaches of the technology have been developed early during Viking, Voyager, Pioneer Venus missions (Marouf et al., 1986; Tyler et al., 1989). Lindal et al. (1987) applied some methods of radio holography for observing multibeam propagation in Uranus atmosphere. They derived the power spectra as a function of time and revealed multibeam propagation caused by a methane cloud layer in the atmosphere of Uranus. Lindal (1992) applied multibeam approach to interpret Voyager Neptune occultation data. However, up to now the phase dependence on

Copy right(C) The Society of Geomagnetism and Earth, Planetary and Space Sciences (SGEPSS); The Seismological Society of Japan; The Volcanological Society of Japan; The Geodetic Society of Japan; The Japanese Society for Planetary Sciences. time in radio occultation measurements has been the main source of information for evaluating the vertical profiles of atmospheric and ionospheric parameters. It may be noted that amplitude data contain valuable information on the refractivity gradient altitude distribution. This information is important for observing wave structures and estimating the parameters of natural phenomena in the upper atmosphere. Attempts to combine phase and amplitude information by applying a backward method have been made by Gorbunov, Gurvich, and Bengtsson (1996), Karayel and Hinson (1997), Mortensen and Hoeg (1998), Mortensen et al. (1999). An attempt to apply a method of deciphering the ray structure in regions of multipath propagation based on diffraction theory and the principles of synthesized aperture has been described by Gorbunov and Gurvich (1998). They provided numerical modeling of the method and shown the possibility for the refinement of the vertical resolution beyond the Fresnel scale up to value $\sim 100 \mathrm{~m}$ at wavelength $20 \mathrm{~cm}$. Radio holographic approach was suggested for this aim also by Pavelyev (1998), Pavelyev et al. (1999), and Hocke et al. (1999) on the basis of the fundamental theoretical radio optics approach derived by Zverev (1975). Main task of both backward propagation and radio holographic methods consists in developing algorithms that using full precision of GPS radio navigational field for achieving extreme accuracy and spatial resolution in evaluation of altitude dependence of meteorological and ionospheric parameters from results of radio occultation measurements.

The application of the radio holographic approach to this 
objective consists in using focused synthetic aperture method. This approach combines trajectory data and the refraction index model to obtain the maximal spatial compression of the main beam separately from the other rays trajectories. This makes possible to evaluate the intensity of radio waves at each beam trajectory and the corresponding angular displacement from a reference beam.

The aim of the present work is to test the radio holographic concept by using the results of radio occultation measurements provided at wavelengths 19 and $24 \mathrm{~cm}$ (GPS/MET data) and $32 \mathrm{~cm}$ ("MIR"-GEO data) and to show possibility of application combined phase and amplitude radio occultation data for observing wave structures in the upper atmosphere.

\section{Radio Holographic Method}

The scheme of radio occultation observation is shown in Fig. 1. The terrestrial atmosphere is modeled locally as being spherically symmetrical, with a local centre of curvature at $O$. One of the "ray paths" followed by radio signals propagating from GPS to LEO satellites (points $G$ and $T$, respectively) lies in the plane of Fig. 1, which also contains the point $O$. The ray has impact parameter $p$ and corresponding refraction angle $\xi(p)$. The next connections are existing between $\xi(p)$ and $p$ for the case of spherical symmetry:

$$
\begin{aligned}
& \beta=\pi+\xi(p)-\theta-\mu-\arcsin \left[p /\left(R_{g}\right)\right], \\
& p=R_{T} \sin (\beta+\mu)
\end{aligned}
$$

where $R_{T}, R_{g}$ is the distances $T O$ and $G O$. Since the functions $\theta(t), R_{T}(t), R_{g}(t)$ are known from trajectory data, Eq. (1) give impact parameter $p$ if the refraction angle $\xi(p)$ or angle $\beta$ are measured. In the case of multibeam propagation the determination of $\xi(p)$ is difficult because the main beam $G T$ is observed on the phone of the sum of the fields of secondary beams that are together with the main beam components of the angular spectrum of radio waves $A_{s}(\beta)$. The angular spectrum presents the radio field $E(\mathbf{r}, t)$ as a superposition of waves having complex amplitude $A_{s j}\left(\beta_{j}\right)$ propagating at different angles $\beta_{j}$ relative to the line $T O$ :

$$
\begin{aligned}
& E(\mathbf{r}, t)=\sum_{j=1}^{M} A_{s j}\left(\beta_{j}\right) \exp \left[i\left(\omega_{o} t-k \Phi\left(\beta_{j}, t\right)\right)\right] \\
& \Phi\left(\beta_{j}, t\right)=\int_{R_{T}}^{R_{g}} n_{j} d l ; \\
& \Phi\left(\beta_{j}, t\right)=S_{b}(t)+S\left(\beta_{j}\right) ; \quad k=2 \pi / \lambda
\end{aligned}
$$

where $\omega_{o}=2 \pi f_{o}, f_{o}$-carrier frequency of radio field, $n(l)$ is the refraction index distribution along $j$-th ray trajectory, $M$ is a number of the ray trajectories connecting points $T$ and $G$ ( $M$ may be a function of time depending on physical conditions in the atmosphere), $\Phi\left(\beta_{j}, t\right)$ is the eikonal defined according to Kravtsov and Orlov (1990). The function $\Phi\left(\beta_{j}, t\right)$ includes the free space $S_{b}(t)$ and combined ionospheric and atmospheric contributions $S\left(\beta_{j}\right)$ to the eikonal. The rays observed at point $T$ may have different origin due to refraction in the atmosphere and ionosphere, scattering on turbulence, diffraction phenomena near caustics, etc. The eikonals $S\left(\beta_{j}\right)$ arising owing to refraction mechanisms have

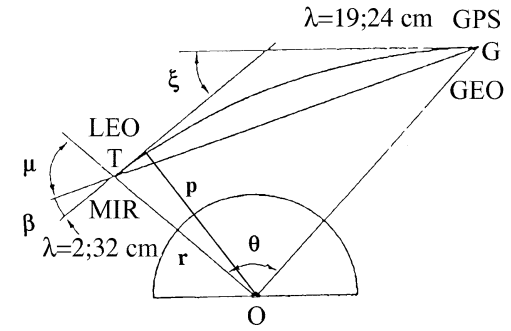

Fig. 1. Scheme of radio occultation observations.

a common property expressed by the next relation derived under assumptions of spherical symmetry of the atmosphere and circular orbits of LEO and GPS satellites (Pavelyev and Yeliseyev, 1989):

$$
F_{d j}=\lambda^{-1} d S\left(\beta_{j}\right) / d t=-\lambda^{-1} p_{j} d \theta / d t
$$

Relation (3) describes connection of Doppler frequency excess $F_{d j}$ with impact parameter $p_{j}$ of $j$-th ray.

Record of complex radio signals along the LEO trajectory is the radio hologram's envelope that contains the amplitude $A(t)$ and phase path excess $\psi(t)=k S_{e}(t)$ of the radio field as functions of time which may be combined in the complex form:

$$
E(t)=A(t) \exp [-i \psi(t)]
$$

Temporal dependencies of the amplitude $A(t)$ and eikonal $S_{e}(t)$ are given in the GPS-MET radio occultation data. Dependence $E(t)$ on time contains information on the spatial distribution of the radio field. The radio field $E(\mathbf{r}, t)(2)$ is equal to $E(t)$ from (4) if $\omega_{o}=0, S_{b}(t)=0$ (the free space term in eikonal is usually removed from radio occultation data) and $\mathbf{r}(t)$ describes the orbital motion of LEO satellite. A reference wave field $E_{m}(t)=\exp \left[i \psi_{m}(t)\right], \psi_{m}(t)=k S_{m}(t)$ may be used to reveal the angular spectra $A_{s j}\left(\beta_{j}\right)$ from radio hologram, where $\psi_{m}(t)$ and $S_{m}(t)$ are the expected phase path excess and eikonal for radio occultation region. The ray corresponding to the phase function $\psi_{m}(t)$ begins in the point $G$ and intersects the direction $T O$ at angle $\beta_{m}(t)$ in the point $T$. The form of the eikonal $S_{m}(t)$ may be different depending on the atmosphere properties according to season, geographic position and expected weather conditions in the radio occultation region. The function $S_{m}(t)$ may be evaluated using various models of the atmosphere and ionosphere, for example, the models proposed by Pavelyev et al. (1996). The next equation may be found after multiplication both sides (2) by the reference field $E_{m}(t)$ :

$$
\begin{aligned}
E(t) E_{m}(t) & =A(t) \exp \left\{-i\left[\psi(t)-\psi_{m}(t)\right]\right\} \\
& =\sum_{j=1}^{M} A_{s j}\left(\beta_{j}\right) \exp \left[-i k\left(S\left(\beta_{j}\right)-S_{m}(t)\right)\right]
\end{aligned}
$$

Using relation (3) the difference $S\left(\beta_{j}\right)-S_{m}(t)$ may be presented inside the time interval $-T / 2<t<T / 2$ in the form (under assumption $d \theta / d t=$ const which is fulfilled for the case of circular orbits of LEO and GPS satellites):

$$
\begin{aligned}
S\left(\beta_{j}\right)-S_{m}(t)= & S_{o j}+d \theta / d t\left[\left(p_{j}-p_{m}\right) t\right. \\
& +d\left(p_{j}-p_{m}\right) / d t t^{2} / 2 !+\cdots \\
& \left.+d^{n-1}\left(p_{j}-p_{m}\right) / d t^{n-1} t^{n} / n !\right],
\end{aligned}
$$


where all derivatives in (6) are corresponding to the time instant $t=0$ that determines the spatial position of the phase center of the focused synthetic aperture. Equation (6) gives the phase distribution along the focused synthetic aperture for $j$-th ray relatively to the reference beam. It is convenient to apply a spectral method for solution (5). For this aim it is necessary to multiply both parts of $(5)$ by factor $\exp (-i \omega t)$, and integrate on time in the interval $-T / 2 \leq t \leq T / 2$. In the result the main power will correspond to the rays that are matched with function $S_{m}(t)$ in the sense that only two terms in the right side of (5) may be retained. For such rays after integration the next equation describing radio hologram's spectrum $W(\omega)$ may be obtained:

$$
\begin{gathered}
W(\omega)=\int_{-T / 2}^{T / 2} d t E(t) E_{m}(t) \exp (-i \omega t) \\
=\sum_{j=1}^{M_{1}} A_{s j}\left(\beta_{j}\right) f\left(\omega, \omega_{j}\right) ; \\
\omega_{j}=k d \theta / d t\left(p_{j}-p_{m}\right)_{o} \\
f\left(\omega, \omega_{j}\right)=\exp \left(-i k S_{o j}\right) \sin X_{j} / X_{j} ; \\
X_{j}=T\left(\omega-\omega_{j}\right) / 2
\end{gathered}
$$

where $M_{1}$ is a number of rays that are in coherence with the reference beam.

According to (8), the module of function $f\left(\omega, \omega_{j}\right)$ has a sharp maximum at value of angle $\beta_{j}$, equal to:

$$
\begin{array}{r}
\beta_{j}=\arcsin \left[\omega / k v+\sin \left(\beta_{m}+\mu_{o}\right)\right]-\mu_{o}, \\
v=R_{T} d \theta / d t
\end{array}
$$

Thus there is correspondence between angle $\beta_{j}$ and the angular frequency $\omega$ described by (9). The measured value of the angle $\beta_{j}$ is corresponding to the time instant $t=0$. According to (7)-(9) the angular spectrum $A_{s j}\left(\beta_{j}(\omega)\right)$ may be found from approximate equation:

$$
\left|A_{s j}\left(\beta_{j}(\omega)\right)\right| \approx|W(\omega)| / \pi,
$$

The angular width of the maximum $\Delta \beta$ depends on $T$. If $T$ increases, the angular resolution in the angular spectrum increases and $\Delta \beta$ diminishes. This is general property of radio hologram: spatial resolution becomes better with increasing radio hologram length. Resulting resolution in the angular spectrum $\Delta \beta$ and corresponding vertical resolution $\Delta h$ can be found from:

$$
\begin{aligned}
& \Delta \beta=0.5 \lambda \Delta f / v, \quad \Delta f=1 / T, \\
& \Delta \beta=\pi /(T k v), \quad \Delta h=L \Delta \beta, \\
& L \approx\left(r^{2}-p^{2}\right)^{1 / 2}
\end{aligned}
$$

where $k=2 \pi / \lambda, T$ is the time of coherent data handling. According to Eq. (11) the accuracy of the radio holographic method increases when $v$ and $T$ are growing and the wavelength is diminishing. The wavelength dependence of angular resolution, Eq. (11), is distinct from the Fresnel one: $\Delta \beta$ (radioholographic) $\sim \lambda$ and $\Delta \beta$ (Fresnel) $\sim \lambda^{1 / 2}$. Due to this difference, the radio holographic method seems to be an effective tool for radio occultation data analysis. The angular position of the main beam $\beta$ according to (1) is related to the impact parameter $p$ and bending angle $\xi(p)$. After estimating dependence $\xi(p)$ the standard Abel inversion procedure may be used for determination the refraction index altitude profile as described by Gorbunov et al. (1996). Hocke et al. (1999) analyzed errors connected with possible influence of the choice of the data window length. It follows from this analysis that the angular resolution of the radio holographic method may achieve 3-5 microradians. The angular spectrum may be prolonged along the rays up to any plane disposed near the atmosphere. In this case the angular spectrum may be interpreted as "radio image" or "radio brightness distribution" of the atmosphere and ionosphere as seen from the LEO orbit.

It may be noted that there exists a deep analogy between the focused synthetic aperture method applied to high resolution radar imaging (see, for example, Wehner (1987), section 6.4 SAR Theory (Focused Aperture)) and the radio holographic approach, except the only difference: a target in the radio occultation case is a ray, moving through the atmosphere. The main requirement in the both cases is to find such function $\psi_{m}(t)$ that gives minimum angular width of the main maximum in the angular spectrum. This may be considered as maximum spatial compression of the radio occultation signal. For fulfilling this requirement the refractivity altitude dependence used in the radio physical model must be close to the real height dependence of the refraction index in the radio occultation region. If the used model of a target is accurate then extreme instrumental spatial resolution may be achieved (one half of the real antenna's aperture in the radar case as it described by Wehner (1987), the angular accuracy $\Delta \beta$ and vertical resolution $\Delta h$ from (11) in the radio occultation case). Using knowledge on the expected atmosphere conditions (included in the phase function $\psi_{m}(t)$ ) for optimal measuring angular position of the ray's in angular spectrum is a main point of the radio holographic method. This is a main distinction from quantum cell approach now under elaboration by Gorbunov et al. (2000). Spectral radio holographic method reveals only the rays in the angular spectrum that are in coherence (or matched in the sense of high resolution radar imaging technology) with the reference beam. Influence of turbulence, horizontal irregularity of the atmosphere and other effects that can't be predicted by reference model diminishes the vertical resolution. Resolution may be lower owing to finite time of ray's existence (in full analogy with time interval of reflection existence from radar target). This time depends on physical mechanism of ray's arising. The amplitude of each ray may depend on time also. In this case radio holographic method may account for the regular time dependence of the ray's amplitude evaluated from knowledge of its physical nature. Elaboration of radio holography methodology for optimal measuring angular position of time depending rays is the task of future work.

\subsection{Revealing multibeam propagation}

Application of the radio holographic approach to preliminary analysis of radio occultation data (MIR/GEO, wavelength $32 \mathrm{~cm}$; GPS/MET wavelength 19 and $24 \mathrm{~cm}$ ) led to direct observation of multibeam propagation in the troposphere. An example of multibeam propagation in the tro- 
posphere (at level $H=3 \mathrm{~km}$ ), obtained from a MIR/GEO radio occultation experiment is shown in Fig. 2 (left side). The time interval between neighbouring spectra was equal to $1 / 32 \mathrm{sec}$, which corresponds to a distance of nearly $250 \mathrm{~m}$ along the LEO trajectory. The time increased from the lower left corner upwards toward the upper right corner. The width of the main beam in the angular spectrum at the half power level was about 40 microradians, which corresponds to the vertical extent of the beam in the troposphere of the order of $80 \mathrm{~m}$. The power in the secondary beam and its vertical size were sometimes of the order of the corresponding values of the main beam. The changes in the angular spectrum were connected with the spatial distribution of the radio field. Usually only one maximum corresponding to the single-ray trajectory connecting transmitter on the MIR orbital station and the receiver on a geo-stationary satellite (GEO) is seen, for example, the spectrum in the left lower corner in Fig. 2. However the gradual transition from one- to three-beam propagation mode is noticeable in Fig. 2. This effect was connected with the intersection of the caustic boundary. Details of this process are important to interpretation in the frame of the fundamental theory of radio wave propagation in a layered atmosphere. The results shown in Fig. 2 give the experimental evidence of the caustic surface intersection in the Earth' atmosphere observed from space and reveal connected diffraction phenomena.

The high vertical resolution of the radio holographic method is also evident from analysis of GPS/MET radio occultation data. The resulting vertical radio brightness distributions are shown in Fig. 2 (right side). This data is related to a radio occultation event on 07 February 1997 (No. 0158). The upper curve shows the experimental angular spectrum at a height of $84 \mathrm{~km}$. The width of the maximum was about 20 microradians and its vertical size was near $60 \mathrm{~m}$. The

\section{VALIDATION OF HIGH SPATIAL RESOLUTION}
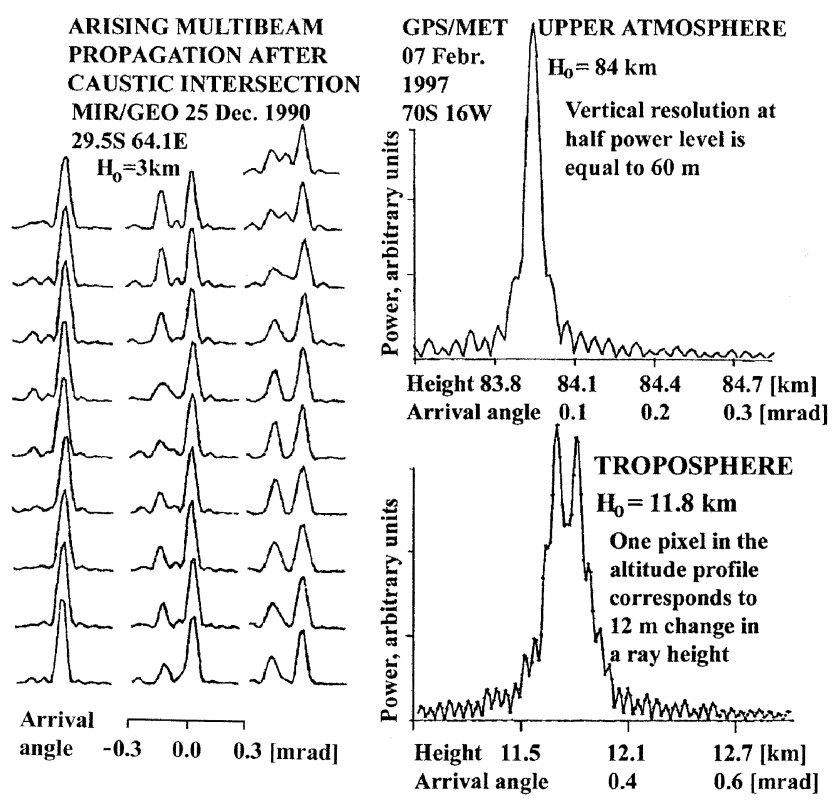

Fig. 2. Evidence of multibeam propagation from data of MIR/GEO and GPS/MET radio occultation experiments. broadening of the angular spectrum in the upper atmosphere may be connected with possible turbulence effects. The radio brightness distribution in the troposphere at a level of $11.8 \mathrm{~km}$ is shown by the lower curve. One pixel in the angular spectrum corresponds to a $0.004-\mathrm{mrad}$ variation in the arrival angle and a $12-\mathrm{m}$ change in the height of the ray perihelion. It follows from Fig. 2 that the radio holographic method may resolve some details in one-dimensional vertical radio images of the atmosphere with a scale of 30-50 meters, which corresponds to a spatial resolution about $1 / 10$ of the Fresnel zone size. This value is some-what higher than the magnitude of the expected spatial resolution about 100 $\mathrm{m}$ corresponding to the modified version of the backward propagation method as described by Mortensen and Hoeg (1998), Mortensen et al. (1999). As it follows from our analysis vertical resolution depends on time of existence of rays in the angular spectrum. This time is different according to mechanism of ray's arising. For the main beam vertical resolution can be made greater $\sim 20-40 \mathrm{~m}$ for the case of the standard atmospheric conditions by choosing larger values of time interval $T$ according to Eq. (11).

\subsection{Comparison of temperature profiles}

To remove the ionospheric part, the bending angle can be linearly corrected by means of interpolation method described by Vorob'ev and Krasil'nikova (1994) and Kursinski et al. (1997). The $\Delta p_{L 1}$ and $\Delta p_{L 2}$ are determined from the position of the major peak in the spectrum of $L 1$ and $L 2$ signals of the same time interval. Then, the ionospheric corrected impact parameter $p$ and bending angle $\xi$ are given by:

$$
\begin{aligned}
& \left.\Delta \xi_{L 1, L 2}=\Delta p_{L 1, L 2}\left[\left(R_{T}^{2}-p_{m}^{2}\right)^{-1 / 2}+\left(R_{g}^{2}-p_{m}^{2}\right)^{-1 / 2}\right)\right] \\
& \xi(p)=\left[f_{1}^{2} \xi_{1}(p)-f_{2}^{2} \xi_{2}(p)\right] /\left(f_{1}^{2}-f_{2}^{2}\right) ; \\
& p_{1,2}=p_{m}+\Delta p_{L 1, L 2} ; \\
& \xi_{1,2}\left(p_{1,2}\right)=\xi_{m}+\Delta \xi_{L 1, L 2}, \quad p=p_{2}
\end{aligned}
$$

where index " $m$ " designates the impact parameter $p_{m}$ and bending angle $\xi_{m}$ connected with $\beta_{m}$ by Eq. (1) and corresponding to chosen model of the atmosphere used for calculation of the reference function $\psi_{m}$.

The temperature profiles were retrieved from the ionospheric corrected bending angle altitude dependence by using Abel inversion, a dry air assumption, hydrostatic equilibrium, and a state equation. Hocke (1997), Schreiner et al. (1999), and Feng and Herman (1999) described details of practical implementation of Abel's inversion. The UCAR data retrievals were used for accuracy checking. The diffraction/multipath effects were reduced in UCAR retrieval by means of backward propagation approach.

In Fig. 3, the temperature difference of both retrievals is shown on the left. In the middle, the absolute temperature profiles are shown (solid line: radio holographic retrieval; dash-dotted line: UCAR retrieval). The vertical gradients of the temperature (e.g., in the tropopause) are mostly accompanied by significant structures of the CASNR signal/noise ratio (SNR of the coarse-acquisition ranging code, $L 1$ wave), which is depicted on the right. The values of the standard deviation between both retrievals were calculated for the selected occultation events for heights below $30 \mathrm{~km}$. The mean difference was $1.6^{\circ}-1.7^{\circ} \mathrm{K}$. The relative large differences be- 
09 Feb.1997 07h 54m 02s $16.0 \mathrm{~S} 112.8 \mathrm{~W} \quad$ 0218-07.23-97.040
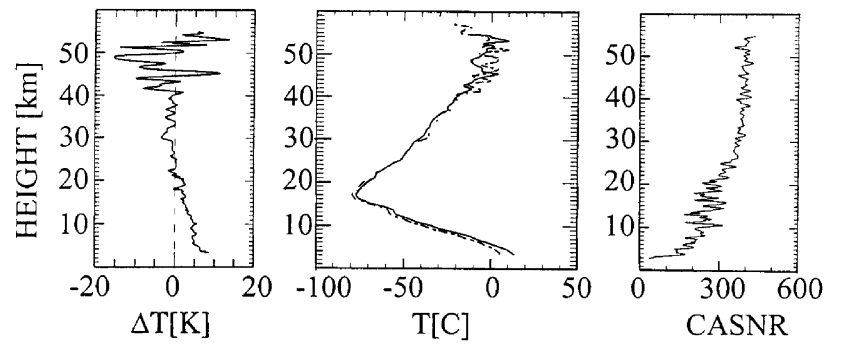

09 Feb. 1997 07h 19m 53s 48.4 N 133.6 E 0206-04.25-97.040
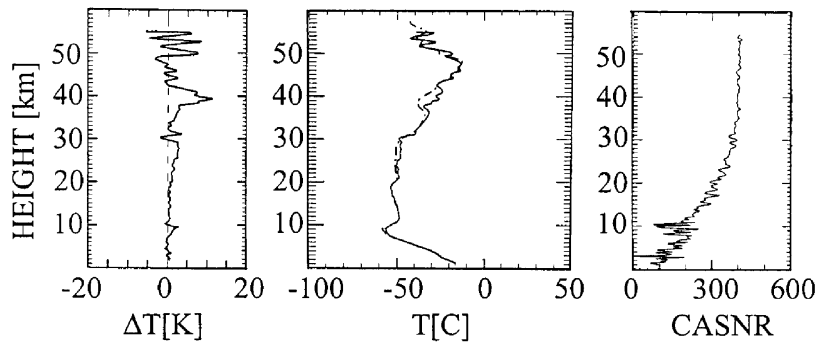

Fig. 3. Comparing temperature profiles (solid lines) with UCAR data.

tween the temperature profiles of our retrieval and the UCAR retrieval in the middle and upper stratosphere (above $35 \mathrm{~km}$ ) were caused by different data filtering as well as by small differences in the upper boundary condition and statistical optimization procedure, which are required for the Abel inversion as shown by Hocke (1997).

\section{Wave's Observation in the Upper Atmosphere}

Processes in the upper atmosphere (hight interval 60-120 $\mathrm{km}$ ) now are staying outside of attention of the most radio occultation works besides some rare publications, for example, Vorob'ev et al. (1999), applied the phase screen theory for deriving electron density distribution in the $E$-layer of ionosphere from amplitude GPS/MET data. However, phenomena in the upper atmosphere can be analyzed more carefully using high-precision GPS/MET radio occultation data. Comparing may be done with results of Earth-based observations for validation, Igarashi et al. (1999). Wave structures in the upper atmosphere are producing short-period changes both in the phase and amplitude radio occultation data at frequencies $L 1$ and $L 2$. The phase changes are hidden under the more intense low-frequency phone arising owing to the ionospheric $F$-layer. The corresponding amplitude variations are visible more clearly, however interference of low-frequency noise must be removed also. In this case the radio holography may be applied in a form of perturbation method that use filtration of low frequency and high frequency components separately of the phase and amplitude radio occultation signal to retrieve electron density altitude profiles and reveal the different kinds of wave motions in the upper atmosphere. For the phase channel it is convenient to use the phase difference $F 1-F 2$ for estimation refractivity profile because existing systematic error in the phases $F 1, F 2$ connected with $2 \pi$ and initial uncertainties in the $F 1, F 2$ data (usually in the raw radio occultation data $F 1_{o}=F 2_{o}=1 \mathrm{~m}$ in the beginning of a measurement session). After removing the low frequency noise (or trends) from the phase and amplitude data (this may be achieved by means of the appropriate model of the upper ionosphere in full analogy with the spectral radio holography approach described above) it is possible to apply two inversion formula separately for the phase and amplitude channels. For the phase channel the Abel's inversion formula may be applied:

$$
\begin{aligned}
& N(h) \approx-C / \pi \int_{a+h}^{\infty} d \kappa(p) /\left[p^{2}-(a+h)^{2}\right]^{1 / 2}, \\
& \kappa(p)=(F 1-F 2)_{r}, \\
& C=f_{1}^{2} f_{2}^{2} /\left[40.3\left(f_{1}^{2}-f_{2}^{2}\right)\right]
\end{aligned}
$$

where $(F 1-F 2)_{r}$ is the phase residuals after subtracting the $F$-layer's trend, $p$ is impact parameter corresponding to free space propagation, $h$ is the height above the Earth's surface, $N(h)$ is the perturbation part of the electron density altitude distribution $\left(\mathrm{el} / \mathrm{m}^{3}\right), C$ is the scaling factor, $f_{1}, f_{2}$ are the frequencies $L 1, L 2[\mathrm{~Hz}]$.

The amplitude variations connected with the ionosphere influence may be used separately for obtaining electron density distribution and its gradient. Influence of the $F$-layer usually corresponds to small long-scale variations in the amplitude that may be not seen on the phone of the changes caused by receiver and transmitter instabilities. The wave variations in the electron density distributions caused shortperiod oscillations in the amplitudes $A_{1}, A_{2}$. Correlation of the intensity variations at two frequency reveals their plasma's origin. For finding gradient of electron density distribution from the amplitude data the equations derived by Pavelyev et al. (1986) may be used:

$$
\begin{gathered}
d N(h) / d h_{1,2} \approx C_{a 1,2} /[(a+h) \pi] \\
\cdot \int_{a+h}^{\infty} p d p d \xi_{1,2}(p) / d p \\
\cdot\left[p^{2}-(a+h)^{2}\right]^{-1 / 2}, \\
C_{a 1,2}=f_{1,2}^{2} / 40.3 \\
d \xi_{1,2}(p) / d p=\left(X_{1,2}-1\right) / X_{1,2} \\
\cdot\left[\left(R_{T}^{2}-p^{2}\right)^{-1 / 2}\right. \\
\left.\left.+\left(R_{g}^{2}-p^{2}\right)^{-1 / 2}\right)\right], \\
X_{1,2}=A_{1,2}^{2} / A_{1,2 o}^{2}
\end{gathered}
$$

where $a$ is the Earth's radius, $p$ is the impact parameter, $A_{1,2}$ is the amplitude of the radio occultation signal, $A_{1,2 o}$ is the amplitude before radio occultation, $C_{a 1,2}$ is a scaling factor depending on frequency. Expressions (14), (15) give the dependence $d N(h) / d h_{1,2}$ on the height $h$ separately for each frequency $f_{1}, f_{2}$. For diminishing influence of the random noise the next formula may be applied for the gradient evaluation:

$$
d N(h) / d h=\left[d N(h) / d h_{1}+d N(h) / d h_{2}\right] / 2
$$

Combined analysis of the results obtained separately from the phase and amplitude data is necessary for removing systematic errors that may arise due to inaccuracies in subtracting of low frequency trends. From radar technology point of view the principal spatial resolution in two independent channels: the phase and amplitude is depending on the length of the 
aperture (or time of observation) as was shown above for the spectral method. This is a main point of radio holographic method in application to the upper atmosphere study.

The results of the application of radio holographic analysis to one GPS/MET occultation event (07 February 1997, No. 0158), $D$-region of the ionosphere, are shown in Fig. 4 . The studied occultation event took place during the local summer daytime in the Antarctic region. For the data given in Fig. 4 the time-spatial coordinates of ray perihelion height $H$ changed from $71.2^{\circ} \mathrm{S} 18.2^{\circ} \mathrm{W}, H=95 \mathrm{~km}, 14 \mathrm{~h} 51 \mathrm{~m} 05 \mathrm{~s} \mathrm{UT}$ to $70.5^{\circ} \mathrm{S} 16.4^{\circ} \mathrm{W}, H=60 \mathrm{~km}, 14 \mathrm{~h} 51 \mathrm{~m} 25 \mathrm{~s} \mathrm{UT}$. Curves 1-3 in Fig. 4 correspond to the experimental phase (curve 1) and amplitude (curve 2 and 3 ) data. Curve 1 describes residuals of phase difference $F 1-F 2$ [m] between carriers $L 1$ and $L 2$ obtained after removing the effect of the upper layers of ionosphere (above $95 \mathrm{~km}$ ). The residual's magnitude was small compared to the general ionospheric contribution and changes within $\pm 1 \mathrm{~cm}$ (less than $1 \%$ of all ionospheric variation in the difference phase path, $F 1-F 2$ ). However, this value is higher, by a factor of 10 , than the noisy phase component, which was nearly $\pm 1 \mathrm{~mm}$ for this event. Curves 2 and 3 show the height dependence of the normalized amplitudes at frequencies $L 1$ and $L 2$ (in logarithmic scale). Comparing the time dependence of the phase-residuals and amplitudes parameters revealed general sensitivity for identifying wave structures in the upper atmosphere. The phase difference is more sensitive to large-scale structures, while the amplitude variations are connected with inhomogeneities having smaller spatial periods. The variations in the amplitude at both frequencies are correlated, and their level is higher at the second frequency. This is evidence of plasma's origin being connected with changes in the electron density altitude profile. Combined analysis of the phase and amplitude data reveals spatial periods from 1-2 $\mathrm{km}$ up to $8-10 \mathrm{~km}$ at altitudes of 72-95 km.

Quantitative conclusions can be drawn under the assumption of spherical symmetry. In this case, the time dependence

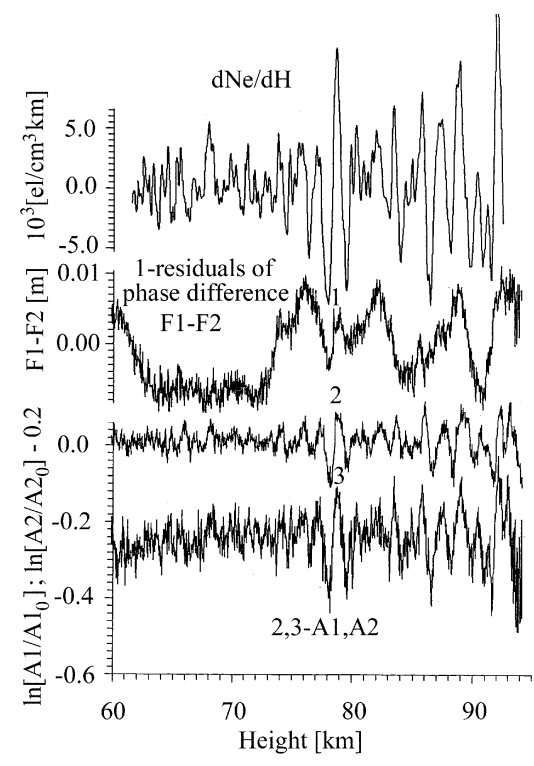

Fig. 4. Retrieved $d N_{e} / d H$ profile. of the amplitude oscillations can be used for revealing the refractivity index gradient and electron density altitude profiles. The obtained $d N_{e} / d H$ profile is shown in Fig. 4 (top). The $d N_{e} / d H$ profile in Fig. 4 revealed wave-like structures in the plasma concentration with a spatial period of $1-2 \mathrm{~km}$ and variations in the electron density gradient from $\pm 5 \cdot 10^{3}$ to $\pm 8 \cdot 10^{3}$ electrons $/\left(\mathrm{cm}^{3} \mathrm{~km}\right)$. The main peak in the gradient is observed at the $H=78 \mathrm{~km}$ with value $11 \cdot 10^{3}$ electrons $/\left(\mathrm{cm}^{3} \mathrm{~km}\right)$. This peak is clearly seen also in the raw amplitude and phase data (feature 1 in Fig. 4). The electron density altitude profile may be obtained by using the dependence $d N_{e} / d H$ on height under the assumption of a certain initial condition, for example, $N_{e}(H)=0$ if $H=60 \mathrm{~km}$.

The results of retrieving the electron density altitude profile $N_{e a}(H)$ obtained by integration from level $H=60 \mathrm{~km}$ using amplitude data are given in Fig. 5 (right curve, amplitude axis). The $N_{e}(H)$ profile can be retrieved from the phase data also. Retrieving $N_{e}(H)$ from the phase data may be achieved by applying Abel's inversion technology to the residual phase altitude dependence (curve 1 in Fig. 4). The results of retrieving $N_{e p}(H)$ dependence are shown in Fig. 5 (left curve). Comparing the $N_{e a}(H)$ and $N_{e p}(H)$ altitude dependencies reveals similar details (marked by numbers and letters in Fig. 5). For example, the 1p local maximum in $N_{e p}(H)$ corresponds to maximum 1a in $N_{e a}(H)$. However, the average magnitudes in $N_{e a}(H)$ and in $N_{e p}(H)$ dependencies are different by a factor of 1.2 to 1.4. This difference may be explained for example by the trend residuals, influence of horizontal gradients etc. As it follows from analysis of the data shown in Figs. 4 and 5 that the combined phase and amplitude radio holographic approach gives high vertical resolution $0.2-0.5 \mathrm{~km}$ (more than the Fresnel's zone size of $1.5 \mathrm{~km}$ ) for revealing wave-like structure in electron density distribution altitude profiles in the $D$ - and $E$-layers of the ionosphere. From physical point of view the resolution is better than standard Fresnel's zone size due to variations of Fresnel's zone dimension in vertical direction owing to wave structures influence. These variations may be seen in correlated phase and amplitude temporal oscillations. Thus radio holographic

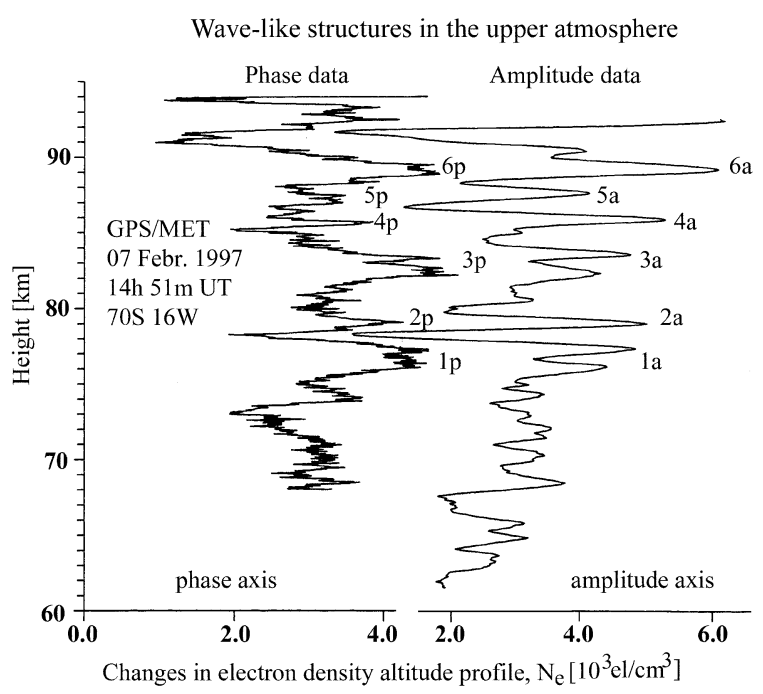

Fig. 5. Results of retrieving $N_{e}(H)$ profile. 
analysis permits to use high-precision GPS/MET radio occultation measurements to identify detailed structures in the electron density profile in the upper atmosphere.

\section{Conclusion}

The main point of radio holographic concept is to introduce radio optics and holographic principles derived in highresolution radar imaging technology into the radio occultation problem. The radio holographic method consists in combining phase and amplitude data to achieve high vertical resolution (more than the Fresnel's zone size) and thereby reveal fine structures in meteorological parameters and electron density altitude profiles with short spatial periods $(0.2-$ $0.5 \mathrm{~km}$ ). As a result extreme values of a vertical resolution (higher than the size of the Fresnel's zone) and more accurate estimates of the parameters of the natural phenomena in the atmosphere and ionosphere may be achieved. The efficiency of the radio holographic method was confirmed by direct observing multibeam propagation and validation of its high spatial resolution using MIR/GEO and GPS/MET radio occultation data.

Acknowledgments. We are grateful to UCAR for access to the GPS/MET data. This contribution was prepared while one of the authors, Dr. A. Pavelyev, was visiting the Communications Research Laboratory, Ministry of Posts and Telecommunications, Japan. The visit was fully supported by TAO, with cooperation of CRL. Dr. A. Pavelyev thus wishes to express his utmost gratitude to TAO and CRL. We are grateful also to Referees A and B for their useful comments.

\section{References}

Feng, D. D. and B. M. Herman, Remotely sensing the Earth's atmosphere using the Global Positioning System (GPS) - the GPS/MET data analysis, Journal of Atmospheric and Ocean Technology, 16, 990-1002, 1999.

Gorbunov, M. E. and A. S. Gurvich, Microlab-1 experiment: Multipath effects in the lower troposphere, J. Geophys. Res., 103, 13,819-13,826, 1998.

Gorbunov, M. E., A. S. Gurvich, and L. Bengtsson, Advanced algorithms of inversion of GPS/MET satellite data and their application to reconstruction of temperature and humidity, Report 211 Max-Planck-Institute for Meteorology ISSN 0937-1060, 1996.

Gorbunov, M. E., S. V. Sokolovskiy, and L. Bengtsson, Space Refractive Tomography of the Atmosphere: Modelling of Direct and Inverse Problems, Report 210 Max-Planck-Institute for Meteorology ISSN 09371060, 1996.

Gorbunov, M. E., A. S. Gurvich, and L. Kornblueh, Comparative analysis of radio holographic methods of radio occultation data, Radio Sci, 35(4), 1025-1034, 2000

Hocke, K., Inversion of GPS meteorology data, Annales Geophysicae, 15, 443-450, 1997.

Hocke, K., A. Pavelyev, O. Yakovlev, L. Barthes, and N. Jakowski, Radio occultation data analysis by radio holographic method, JASTP, 61, 11691177, 1999.

Igarashi, K., Y. Murayama, K. Hocke, R. Yamazaki, M. Kunitake, M. Nagayama, and I. Nishimuta, Coordinated observations of the dynamics and coupling processes of mesosphere and lower thermosphere winds with MF radars at the middle-high latitude, Earth Planets Space, 51, 657-664, 1999.

Karayel, E. T. and D. P. Hinson, Sub-Fresnel-scale vertical resolution in atmospheric profiles from radio occultation, Radio Sci., 32(2), 411-428, 1997.

Kravtsov, Yu. and Yu. N. Orlov, Geometrical Optics of Inhomogeneous Media, Springer-Verlag, New York, 1990.

Kursinski, E. R., et al., Initial results of radio occultation observations of Earth's atmosphere: using the Global Positioning System, Science, 271, 1107-1110, 1996.
Kursinski, E. R., G. A. Hajj, J. T. Schofield, R. P. Linfield, and K. R. Hardy, Observing Earth's atmosphere with radio occultation measurements using the Global Positioning System, J. Geophys. Res., 102(D19), 23,42923,465, 1997.

Lindal, G. F., The atmosphere of Neptune: An analysis of radio occultation data acquired with Voyager 2, Astron. J., 103, 967-982, 1992.

Lindal, G. F., J. R. Lyons, D. N. Sweetnam, V. R. Eshleman, D. P. Hinson, and G. L. Tyler, The atmosphere of Uranus: results of radio occultation measurements with Voyager 2, J. Geophys. Res., 92, 14,987-15,001, 1987.

Marouf, E. A., G. L. Tyler, and P. A. Rosen, Profiling Saturn rings by radio occultation, Icarus, 68, 120-166, 1986.

Melbourne, W. G., E. S. Davis, C. B. Duncan, G. A. Hajj, K. R. Hardy, E. R. Kursinski, T. K. Meehan, L. E. Young, and T. P. Yunck, The Application of Spaceborne GPS to Atmospheric Limb Sounding and Global Change Monitoring, 147 pp., JPL Publication 94-18, April 1994.

Mortensen, M. D. and P. Hoeg, Inversion of GPS occultation measurements using Fresnel diffraction theory, Geophys. Res. Lett., 25(13), 2441-2449, 1998 .

Mortensen, M. D., R. P. Linfield, and E. R. Kursinski, Vertical resolution approaching $100 \mathrm{~m}$ for GPS occultations of the Earth's atmosphere, Radio Sci., 34(6), 1475-1483, 1999.

Pavelyev, A., On the possibility of radio holographic investigation on communication link satellite-to-satellite, Radioteknika i elektronika, 43(8), 939-944, 1998 (in Russian).

Pavelyev, A. and S. D. Yeliseyev, Study of the Atmospheric Layer near the Ground using Bistatic Radar, Journal of Communication Technology and Electronics, No. 9, 124-130, 1988.

Pavelyev, A., S. Matugov, I. Kalashnikov, and O. Yakovlev, Analysis of the features of radio occultation method for the Earth's atmosphere study, in Electromagnetic Waves in the Atmosphere and Space, pp. 208-218, "Nauka" Ed., Moscow, 1986 (in Russian).

Pavelyev, A., A. V. Volkov, A. I. Zakharov, S. A. Krytikh, and A. I. Kucherjavenkov, Bistatic Radar as a Tool for Earth Investigation Using Small Satellites, Acta Astronautica, 39, 721-730, 1996

Pavelyev, A., K. Hocke, O. Yakovlev, N. Jakowski, J. Wickert, S. Matugov, A. Kucherjavenkov, A. Wehrenpfennig, and A. Zakharov, Radio holographic method for atmosphere sounding using small satellite, in Small satellites for Earth Observation. Digest of the 2-nd International Symposium of the International Academy of Astronautics, edited by H. P. Roser, R. Sandau, A. Valenzuella, pp. 199-202, Wissenschaft und Technik Verlag, Berlin, 1999.

Rocken, C., et al., Analysis and validation of GPS/MET data in the neutral atmosphere, J. Geophys. Res., 102(D25), 29,849-29,866, 1997.

Schreiner, W. S., S. V. Sokolovskij, C. Rocken, and D. C. Hunt, Analysis and validation of GPS/MET radio occultation data in the ionosphere, Radio Sci., 34(4), 949-966, 1999.

Tyler, G. L., et al., Voyager radio science observations of Neptune and Triton, Science, 246, 1466-1473, 1989.

Vorob'ev, V. V. and T. G. Krasil'nikova, Estimation of accuracy of the atmosphere refractive index recovery from Doppler shift measurements at frequencies used in the NAVSTAR system, Izv. Russ. Acad. Sci., Physics of the Atmosphere and Ocean, Engl. Transl., 29(5), 602-609, 1994.

Vorob'ev, V. V., A. S. Gurvich, V. Kan, S. V. Sokolovskiy, O. V. Fedorova, and A. V. Shmakov, Structure of the Ionosphere from the RadioOccultation GPS-“Microlab-1' Satellite Data: Preliminary Results, Earth Observations and Remote Sensing, 15, 609-622, 1999.

Ware, R., et al., GPS sounding of the atmosphere from low Earth orbitPreliminary results, Bull. Am. Meteorol. Soc., 77, 19-40, 1996.

Wehner, D. R., High Resolution Radar, 573 pp., Ed. Artech House, Inc. 685 Canton Street Norwood, MA 02062, 1987.

Yakovlev, O. I., Space Radio Physics, edited by By Russian Fund of Fundamental Researches (RFBR), 478 pp., Moscow, 1998 (in Russian).

Yakovlev, O. I., S. S. Matyugov, and I. A. Vilkov, Attenuation and scintillation of radio waves in the Earth's atmosphere from radio occultation experiments on satellite-to-satellite links, Radio Sci., 30(3), 591-602, 1995

Zverev, V. A., Radio Optics, edited by Soviet Radio, Moscow, 1975 (in Russian).

K. Igarashi (e-mail: igarashi@crl.go.jp), A. Pavelyev (e-mail: agp117@ ire216.msk.su), K. Hocke (e-mail: Hocke@gfz-potsdam-de), D. Pavelyev, I. A. Kucherjavenkov, S. Matyugov, A. Zakharov, and O. Yakovlev 